\title{
Pilot study of totally thoracoscopic periareolar approach for minimally invasive mitral valve surgery. Towards even less invasive?
}

\author{
Marcin Maruszewski ${ }^{1}$, Radosław Smoczyński ${ }^{1}$, Mariusz Kowalewski ${ }^{1}$, Maciej Bartczak ${ }^{1}$, Anna Witkowska ${ }^{1}$, \\ Jakub Staromłyński ${ }^{1}$, Dominik Drobiński ${ }^{1}$, Mariusz Kujawski ${ }^{1}$, Piotr Suwalski ${ }^{1,2}$ \\ ${ }^{1}$ Department of Cardiac Surgery, Central Clinical Hospital of the Ministry of Interior, Warsaw, Poland \\ ${ }^{2}$ Department of Cardiac Surgery, Centre of Postgraduate Medical Education, Warsaw, Poland
}

Videosurgery Miniinv 2019; 14 (2): 326-332

DOI: https://doi.org/10.5114/wiitm.2019.81663

\begin{abstract}
Introduction: Minimally invasive mitral valve surgery (MIMVS) has become a widely accepted alternative to the standard sternotomy approach for treatment of mitral valve (MV) disease. Because the extent and location of mini-thoracotomies employed for MIMVS vary from center to center, the conclusions regarding superior cosmesis are not generalizable. The totally thoracoscopic periareolar (TTP) - MIMVS technique has been used at our department for minimally invasive cardiac surgery since 2015.

Aim: To report early surgical data as well as mid-term outcomes in patients undergoing TTP-MIMVS.

Material and methods: Between 2015 and 2017, 48 consecutive patients (mean age: 65.4 \pm 10 ; 83\% men; EuroSCORE II: $5.1 \pm 4 \%$ ) underwent TTP-MIMVS due to mitral and mitral/tricuspid valve (TV) disease; patients' demographics and clinical outcomes were prospectively collected. Kaplan-Meier estimates of survival and freedom from re-intervention were analyzed as well.

Results: Mean follow-up was 1.7 (max 2.5) years. Of 48 patients, 33 (69\%) underwent isolated MV repair, 4 (8\%) isolated MV replacement and 11 (23\%) MV/TV repair. The cardiopulmonary bypass and aortic cross-clamp time was $166 \pm 70$ and $103 \pm 39$ min respectively. There was no conversion to either full sternotomy or a mini-thoracotomy approach. Median (interquartile range) duration of intensive care unit stay was 1.2 (1.0-2.0) days. There was one in-hospital death (2.1\%) in the TTP-MIMVS group. No strokes or wound infections were observed. Within the investigated follow-up, the freedom from reoperation rate was 96.4\%; remote survival was estimated at $96.9 \%$.

Conclusions: The study proved that TTP-minimally invasive surgery was safe and feasible in mitral and tricuspid valve surgery. It has been associated with superior esthetics. Mitral repairs performed through TTP access are durable in mid-term observation.
\end{abstract}

Key words: minimally invasive surgery, mitral valve, extracorporeal circulation, periareolar access, valvular disease.

\section{Introduction}

Over the last 20 years, minimally invasive mitral valve surgery (MIMVS) has established itself as an alternative to the conventional sternotomy approach and has increasingly been used in patients with mitral valve pathology. Rather than a single approach, MIMVS refers to a collection of techniques and operation-specific technologies such as modified perfusion and visualization techniques that are aimed at minimizing surgical access and trauma. 
Several studies have reported promising outcomes with MIMVS as compared to conventional surgery with less pain, shorter hospital stays, faster return to normal activities, potential cost savings and superior cosmesis [1-4]. Yet, because the extent and location of mini-thoracotomies employed vary from center to center, the conclusions regarding superior esthetics with minimally invasive surgery are not generalizable [5-7].

Recently, some totally thoracoscopic techniques have been introduced, with excellent results [8-10]. The totally thoracoscopic periareolar (TTP) access employs an incision through the natural scarring in the body around the right nipple. This new approach seems to be comparable or even superior to the minithoracotomy access with regards to preferable esthetic results [11].

\section{Aim}

The aim of the current paper was to report early surgical data as well as mid-term outcomes in patients operated on because of MV disease with totally thoracoscopic periareolar access.

\section{Material and methods}

\section{Study population}

The study involved 48 consecutive patients undergoing heart surgery between 2015 and 2017 due to mitral valve disease. Data were collected prospectively in a single-center- and all-participant registry. After completion, these were retrospectively analyzed. A primary anatomical exclusion criterion for the TTP approach was the female gender, since this technique required an incision around the right nipple and the preparation of the tissue around the mammary gland; in fact, that was revised shortly after and women with a small mammary gland were also considered for the TTP approach. Patients with endocarditis and re-do procedures were excluded.

\section{Periareolar approach. Surgical technique}

A standard peripheral cannulation for extracorporeal circulation (ECC) in MIMVS was advocated. Cannulation of the femoral vessels was performed through surgical access (via a 3-4 cm groin incision) using Seldinger's technique. For venous cannulation, a 22-25 Fr and $60 \mathrm{~cm}$ long, perforated soft cannula (VFEM Femoral Venous Cannula, Edwards Lifesciences, USA or RAP cannula, LivaNova, United Kingdom) was used. The positioning of the venous cannula with its tip in the superior vena cava was achieved under transesophageal echocardiography (TEE) guidance. In patients scheduled for elective tricuspid valve intervention, an additional venous cannula (EOPA Medtronic, USA or OptiSite, Edwards Lifesciences, USA) was inserted into the internal jugular vein. The femoral artery was cannulated with a 16-18 Fr OptiSite (Edwards Lifesciences, USA) cannula. Choice of the cannulas was left to the sur-
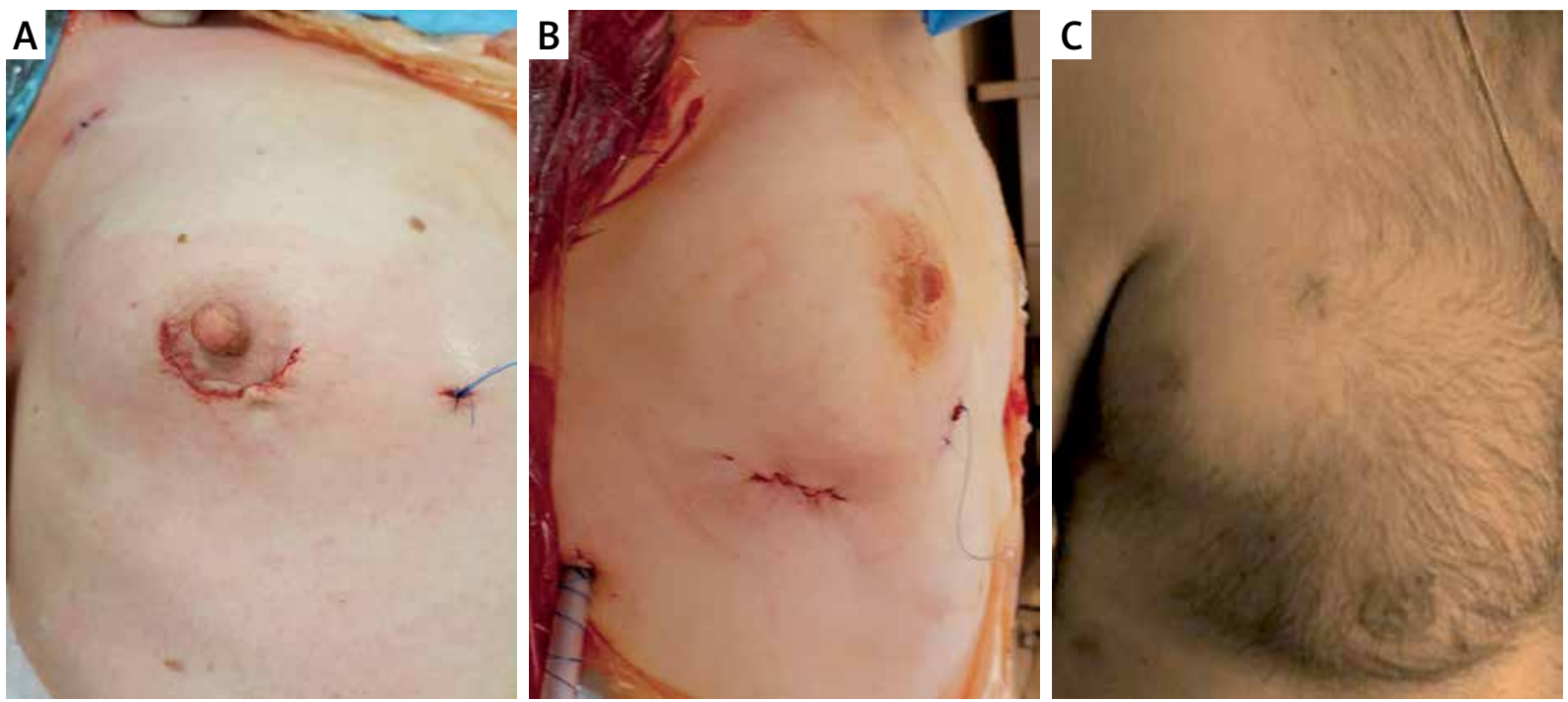

Photo 1. Comparison of the skin incision in the totally thoracoscopic and the minithoracotomy procedures (A, B) and 3 months after surgery in a male patient (C) 


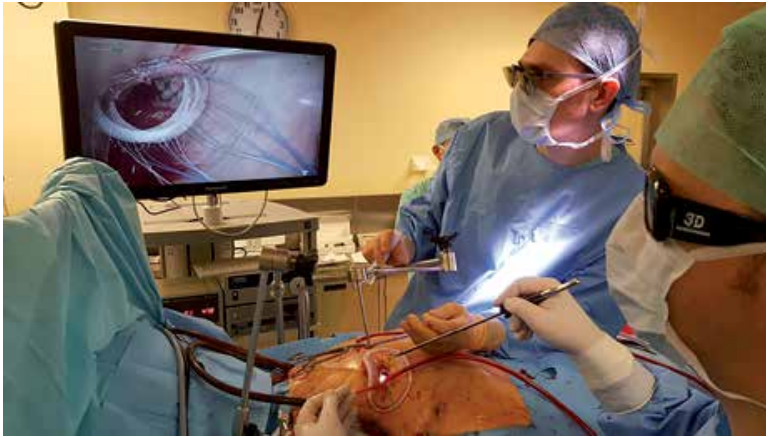

Photo 2. Mitral valve repair using the 3D vision totally thoracoscopic periareolar technique

geons' preference. A right lateral chest wall incision of $3.5-4.0 \mathrm{~cm}$ around the bottom of the nipple was performed (Photos $1 \mathrm{~A}-\mathrm{C}$ ); two additional $1.0 \mathrm{~cm}$ incisions were made for the thoracoscope and the aortic clamp in the $2^{\text {nd }}$ and $3^{\text {rd }}$ intercostal spaces. The chest cavity was inflated with carbon dioxide at a flow rate of $1.5-2 \mathrm{l} / \mathrm{min}$. In all patients, a Chitwood clamp was used for cross clamping of the aorta. The heart was arrested with either Bretschneider or $4: 1$ cold blood cardioplegia infused into the aortic root under direct vision. Mitral annuloplasty was performed using semirigid mitral rings (Carpentier-Edwards Physio or Physio II Annuloplasty Ring, Edwards Lifesciences, USA or Memo 3D LivaNova, United Kingdom) (Photo 2). In patients with mitral valve leaflet prolapse, a correction was performed using

Table I. Preoperative characteristics and indications for surgery

\begin{tabular}{|lc|}
\hline Parameter & TTP-MIMVS $(n=48)$ \\
\hline Age [years] & $65.4 \pm 10$ \\
\hline Male, $n(\%)$ & $40(83)$ \\
\hline COPD, $n(\%)$ & $2(4)$ \\
\hline Diabetes, $n(\%)$ & $8(17)$ \\
\hline LVEF (\%) & $52 \pm 14$ \\
\hline EuroSCORE II & $5.1 \pm 4.0$ \\
\hline NYHA II class & $78.2 \%$ \\
\hline Isolated MV regurgitation, $n(\%)$ & $33(69)$ \\
\hline Isolated MV stenosis, $n(\%)$ & $4(8)$ \\
\hline Secondary TV regurgitation, $n(\%)$ & $11(23)$ \\
\hline
\end{tabular}

COPD - chronic obstructive pulmonary disease, LVEF - left ventricle ejection fraction, MV - mitral valve, TV-tricuspid valve. the polytetrafluoroethylene (PTFE) loop technique. In the case of concomitant tricuspid valve surgery, an annuloplasty was performed on the arrested heart with a dedicated ring (MC3 Tricuspid Annuloplasty Ring, Edwards Lifesciences, USA). The acute result of the mitral and tricuspid valves' repair was confirmed in TEE. Remnant regurgitation less than or equal to $1+$ was accepted.

\section{Statistical analysis}

A statistical analysis was performed using the Statistica program (StatSoft, Inc. 2012). The Shapiro-Wilk W-test was used to test for normality. When the $W$ statistical value proved significant, the hypothesis that the corresponding distribution is normal was rejected. Normally distributed continuous variables were expressed as a mean \pm standard deviation or median and interquartile range (IQR). Nonparametric and parametric data correlations were evaluated using either the Spearman rank test or the Pearson test. Logistic regression was fitted to identify the mortality and reoperation risk factors. The Kaplan-Meier curves were used for presentation of overall survival and freedom from reoperation.

\section{Results}

\section{Preoperative characteristics}

During the study period 2015-2017, 98 minimally invasive mitral/tricuspid procedures were performed; of those 48 were conducted via the TTP approach. Study subjects were predominantly male (83.3\%); $p<0.001$ aged $65.4 \pm 10$ years. The principal indications were MV regurgitation in 44 (91.7\%) and MV stenosis in 4 (8.3\%); additional TV regurgitation was present in 11 (22.9\%). Mean EuroSCORE II was $5.1 \pm 4.0$ and ranged from 0.2 to 14.4 , primary MV regurgitation was diagnosed in 38 (86.4\%), secondary MV regurgitation in $6(13.6 \%)$ patients. Predominant posterior leaflet prolapse was the most frequent regurgitation cause $31 / 48$ (81.6\%), followed by anterior leaflet prolapse. Detailed characteristics are presented in Table I.

\section{Surgical characteristics}

Mitral valve repair was the preferred approach in $44(91.7 \%)$ cases, followed by MV replacement in 4 (8.3\%); the PTFE loops and annuloplasty rings were used in all MV repair cases. Replaced valves were bi- 
oprostheses in all 4 cases. No procedural complications occurred. A conversion to full sternotomy was not required in any patient. No conversion from a totally thoracoscopic approach to the video-assisted minithoracotomy approach was needed either. Mean aortic cross-clamp time was $103 \pm 39$ min while total ECC time was $166 \pm 53 \mathrm{~min}$ (Table II); average blood loss was $445 \pm 308 \mathrm{ml}$ with a median (IQR) number of transfused blood units per patient of 1.0 (0-2.0). Two patients required chest revision due to increased postoperative bleeding. Mechanical circulatory support (i.e. by means of extracorporeal membrane oxygenation, an intra-aortic balloon pump or a left ventricular assist device) was not necessary in any patient. The average postoperative mechanical ventilation time was $7.6 \pm 8.0 \mathrm{~h}$; extended mechanical ventilation (over $24 \mathrm{~h}$ ) was needed in 1 (2.1\%) patient. Length of ICU stay was 1.2 (1.0-2.0) days. Wound infection requiring surgical intervention did not occur in any patients operated on using the totally endoscopic technique (Table II). One patient died on post-op day 14, due to multiorgan failure; he underwent an MV and TV combined procedure and his EuroSCORE II was > 14. No strokes occurred.

Freedom from reoperation was estimated at $96.4 \%$ within the investigated follow-up (Figure 1). The overall estimated survival was $96.9 \%$ (Figure 2). Risk factors for mortality and reoperation are presented in Table III. Among them, length of ICU stay $(p=0.003)$ and number of transfusions $(p=0.05)$ were associated with increased mortality. Surgical access, operation type, baseline patients' and proce-

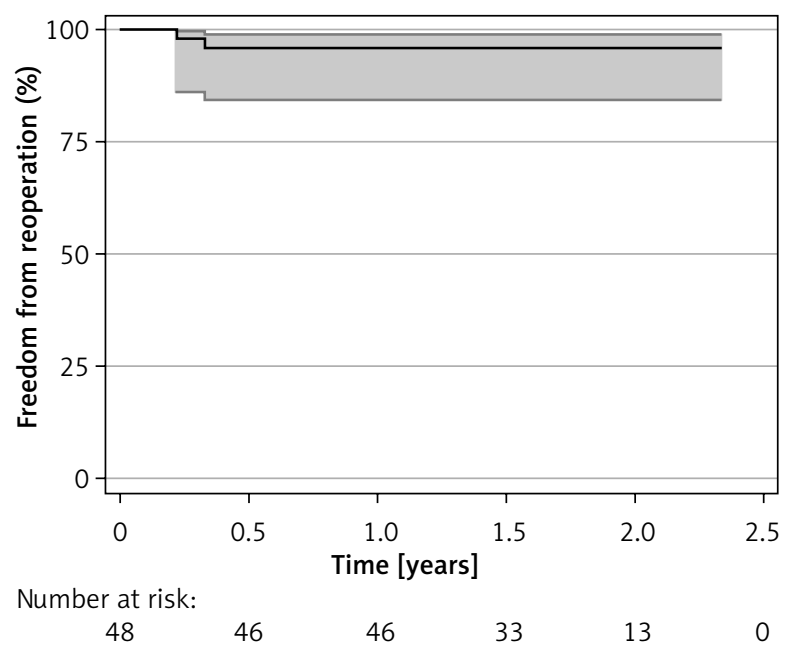

Figure 1. Kaplan-Meier estimated freedom from reoperation
Table II. Clinical outcomes

\begin{tabular}{|c|c|}
\hline Parameter & Value \\
\hline Mortality & $1(2.1 \%)$ \\
\hline Conversion to full sternotomy & 0 \\
\hline Aortic cross-clamp time [min] & $103 \pm 39$ \\
\hline ECC time $[\mathrm{min}]$ & $162 \pm 53$ \\
\hline Chest revision for bleeding & $2(4.2 \%)$ \\
\hline ECMO/IABP/LVAD & 0 \\
\hline Wound infection & 0 \\
\hline Stroke/TIA & 0 \\
\hline Mechanical ventilation time $[\mathrm{h}]$ & $8 \pm 42$ \\
\hline Extended mechanical ventilation (over $24 \mathrm{~h}$ ) & $1(2.1 \%)$ \\
\hline ICU stay, median (IQR) [days] & $1.2(1.0-2.0)$ \\
\hline Total drainage [ml] & $445 \pm 308$ \\
\hline Number of RBC units transfused, median (IQR) & $1.0(0-2.0)$ \\
\hline
\end{tabular}

dural characteristics were not associated with either risk of mortality or reoperation.

\section{Discussion}

In the last two decades, mitral valve surgery has significantly evolved [1-9]. Key progress has been made due to the introduction of the combination of minimally invasive techniques and standardized valve repair techniques [12-16]. The benefits of minimally

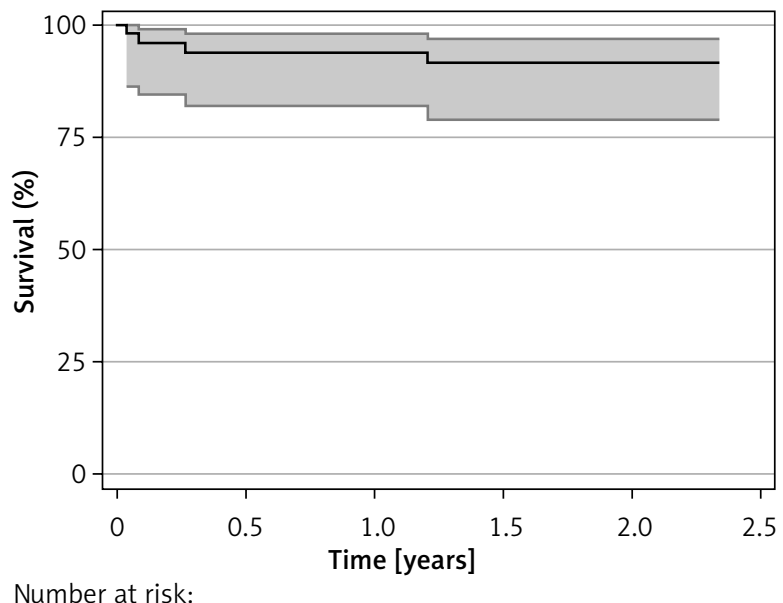

$\begin{array}{lllll}48 & 45 & 45 & 32 & 13\end{array}$

0

Figure 2. Kaplan-Meier estimated survival 
Table III. Mortality and reoperation risk factors

\begin{tabular}{|lcccc|}
\hline Variable & \multicolumn{2}{c}{ Mortality } & Reoperation \\
\cline { 2 - 5 } & OR $(95 \% \mathrm{Cl})$ & $P$-value & OR $(95 \% \mathrm{Cl})$ & $P$-value \\
\hline Surgical access & $1.26(0.31-5.13)$ & 0.75 & $1.45(0.12-6.71)$ & 0.76 \\
\hline Operation type & $0.97(0.36-2.59)$ & 0.94 & $0.54(0.08-3.43)$ & 0.51 \\
\hline Gender & $1.05(0.28-3.89)$ & 0.93 & $1.06(0.14-1.42)$ & 0.98 \\
\hline Age & $0.98(0.93-1.03)$ & 0.58 & $1.05(0.93-1.19)$ & 0.36 \\
\hline LVEF & $0.96(0.94-0.99)$ & 0.20 & $0.97(0.92-1.01)$ & 0.14 \\
\hline Diabetes & $3.56(0.93-13.7)$ & 0.06 & $2.44(0.21-28.4)$ & 0.47 \\
\hline COPD & $1.73(0.2-15.2)$ & 0.62 & $0.04(0.00-0.3)$ & 0.77 \\
\hline EuroSCORE II & $1.09(1.01-1.18)$ & 0.03 & $1.05(0.91-1.22)$ & 0.45 \\
\hline ECC time & $1.01(0.99-1.02)$ & 0.50 & $1.00(0.99-1.01)$ & 0.21 \\
\hline Aortic cross-clamp time & $1.00(0.99-1.02)$ & 0.94 & $1.00(0.98-1.02)$ & 0.67 \\
\hline Total drainage & $1.00(0.99-1.01)$ & 0.23 & $0.98(0.98-1.01)$ & 0.56 \\
\hline Transfusions & $1.25(0.99-1.58)$ & 0.05 & $0.84(0.39-1.8)$ & 0.65 \\
\hline Mechanical ventilation time & $1.00(0.99-1.02)$ & 0.11 & $0.99(0.92-1.05)$ & 0.81 \\
\hline ICU stay & $1.18(1.05-1.32)$ & 0.003 & $0.98(0.71-1.36)$ & 0.93 \\
\hline
\end{tabular}

ECC - extracorporeal circulation, ICU - intensive care unit, COPD - chronic obstructive pulmonary disease, LVEF - left ventricle ejection fraction.

invasive surgery in the mitral valve setting have been summarized in the consensus statement of the International Society of Minimally Invasive Cardiothoracic Surgery (ISMICS) [17]; these include the reduction of blood loss, the reduced need of transfusion, a lower risk of postoperative atrial fibrillation and a shortened length of hospital stay. The downsides observed were longer aortic cross-clamp and ECC time, which however were shown not to have any significant effect on postoperative complications. Moreover, several reports showed improved results of the minimally invasive approach in comparison to the standard sternotomy access in patients with increased operative risk, such as chronic kidney disease or undergoing redo surgery $[18,19]$. Numerous studies from leading cardiac surgery clinics have shown the effectiveness and durability of mitral repair performed using minimally invasive techniques [20], suggesting however volume effect. Regardless, a minimally invasive program for mitral valve surgery is being successfully introduced in a growing number of hospitals [21], even despite an evident necessity of additional training and financial resources.

The most widely performed minimally invasive setting is the video-assisted approach through right minithoracotomy and femoral vessels' cannulation [22]. The periareolar totally thoracoscopic access gained some popularity recently as potentially being an even less invasive method [11]. The current pilot study of the TTP approach for minimally invasive surgery has shown promising results as well. Our findings therefore endorse the endoscopic approach as the next step in the evolution of minimally invasive mitral valve surgery.

Several issues, however, need to be addressed concerning our results. Firstly, the totally endoscopic technique has an anatomic limitation; we found that this approach can safely be used in male patients; however, in our opinion it should carefully be considered in females since it requires mammary gland preparation. Exceptions may be made for selected females with small mammary glands. Moreover, in males, the position of the thoracotomy incision was determined by the position of the nipple and was typically located more anteriorly and, at times, also superiorly when compared to video-assisted access. Consequently, safe introduction of the totally endoscopic mitral valve surgery program requires of the surgeon to have a combined experience in 'direct vision' minimally invasive valve surgery, mitral valve 
repair and thoracoscopic procedures. It should be stressed that the main technical challenge for the surgeon while learning the endoscopic technique is the change from having direct vision to total video surgery in crucial steps of the procedure.

Several previous studies reported increased risk of cerebral complications during retrograde arterial perfusion in minimally invasive mitral valve procedures [23]; others pointed to complications during aortic occlusion [24]; however, the use of cerebral oximetry guided extracorporeal circulation along with TEE as a routine practice in our clinic resulted in a significant reduction of stroke risk in the abovementioned circumstances. The proposed strategy is based on zero tolerance for significant changes in brain oxygenation during the cardiopulmonary bypass and the adjustment of immediate perfusion parameters and cannulas' position. Cerebral desaturation during operations is a proven predictor of cognitive decline and should, therefore, be avoided at all costs [25]. In our series, no in-hospital stroke occurred.

Worth mentioning is the standardized perfusion protocol of moderate hypothermia (between $32^{\circ} \mathrm{C}$ and $34^{\circ} \mathrm{C}$ ) and oxygen delivery (goal directed perfusion protocol) $[26,27]$. Another practice is defining patients at high risk of stroke in preoperative CT scans, which could help in peripheral cannulation planning and aorta clamping (Endoclamp vs transthoracic clamp) [24, 28, 29].

The future place of the totally endoscopic approach in cardiac surgery needs to be discussed with respect to robotic techniques [30]. It may seem that the totally thoracoscopic technique presents several advantages over robotic surgery. Firstly, the number of surgical incisions is vital in both thoracoscopic and the robotic approaches. In our technique, the following surgical cuts are performed: access to femoral vessels (this can be performed percutaneously), one working port, one camera port and one port for the aortic clamp placement (if needed). According to the latest studies from Musumeci et al. [12], the periareolar robotic approach results in a similar total [mm] length of the incision. Secondly, when compared to the robotic access, the thoracoscopic approach was associated with shorter aortic cross clamp time, lower costs and a shorter learning curve for the surgeon. Last but not least, the introduction of the totally endoscopic mitral valve surgery might be easier than that of the robotic surgery program even for experienced surgeons. The economic aspect is also not to be overlooked [31].

The use of stereoscopic vision systems is a clear advantage of robotic surgery; however, the latest 3D thoracoscopic systems in clinical observations have already greatly facilitated the totally thoracoscopic surgery. In our experience, the current 3D optics provide the surgeon with a better sense of the working space, especially with regard to the subvalvular apparatus. The 3D optics facilitate the performance of complex and precise movements, which necessitate both eyeballing and actual distance assessment, especially when it comes to examining the structures positioned parallel to the surgeon's axis of vision, e.g. measuring the proper length of PTFE loops or chordae. Using 3D optics may also, in fact, provide better comfort of reoperation through minimally invasive access. The downside of the use of $3 D$ optics in cardiac surgery is the inability to change the camera's viewing angle without the surgeon making a movement of the entire 3D camera arm, while other camera devices require only the touching of a small switch to change the viewing angle.

Several limitations to the current study need to be acknowledged. Firstly, the study was designed as an analysis of results from a single center and is thus based on the case study of a limited group of patients operated on using the new technique, and thus includes the team's learning curve, which may not be a factor present in other circumstances. Secondly, the number of patients included in the totally endoscopic approach group is limited and has a gender preference due to the very nature of the incision necessary in this approach.

\section{Conclusions}

The study proved that TTP-minimally invasive surgery was safe and feasible in mitral and tricuspid valve surgery. It has been associated with superior esthetics. Mitral repairs performed through TTP access are durable in mid-term observation.

\section{Conflict of interest}

The authors declare no conflict of interest.

\section{References}

1. Modi P, Hassan A, Chitwood WR Jr. Minimally invasive mitral valve surgery: a systematic review and meta-analysis. Eur J Cardiothorac Surg 2008; 34: 943-52. 
2. Walther T, Falk V, Metz S, et al. Pain and quality of life after minimally invasive versus conventional cardiac surgery. Ann Thorac Surg 1999; 67: 1643-7.

3. Cohn LH, Adams DH, Couper GS, et al. Minimally invasive cardiac valve surgery improves patient satisfaction while reducing costs of cardiac valve replacement and repair. Ann Surg 1997; 226: 421-6, discussion 427-8.

4. Lapenna E, Torracca L, De Bonis M, et al. Minimally invasive mitral valve repair in the context of Barlow's disease. Ann Thorac Surg 2005; 79: 1496-9.

5. Qiu Z, Chen X, Xu Y, et al. Does full sternotomy have more significant impact than the cardiopulmonary bypass time in patients of mitral valve surgery? I Cardiothorac Surg 2018; 13: 29.

6. Algarni KD, Suri RM, Daly RC. Robotic-assisted mitral valve repair: surgical technique. Multimed Man Cardiothorac Surg 2014; 2014: pii: mmu022. doi: 10.1093/mmcts/mmu022.

7. Ritwick B, Chaudhuri K, Crouch G, et al. Minimally invasive mitral valve procedures: the current state. Minim Invasive Surg 2013; 2013: 679276.

8. Zhao G, Gao J, Liu Y, et al. Two-incision totally thoracoscopic approach for mitral valve replacement. Int Heart J 2017; 58: 894-9.

9. Casselman FP, Van Slycke S, Dom H, et al. Endoscopic mitral valve repair: feasible, reproducible, and durable. J Thorac Cardiovasc Surg 2003; 125: 273-82.

10. Suwalski P, Witkowska A, Drobiński D, et al. Stand-alone totally thoracoscopic left atrial appendage exclusion using a novel clipping system in patients with high risk of stroke - initial experience and literature review. Kardiochir Torakochir Pol 2015; 12: 298-303.

11. Poffo R, Montanhesi PK, Toschi AP, et al. Periareolar access for minimally invasive cardiac surgery: the Brazilian technique. Innovations (Phila) 2018; 13: 65-9.

12. Musumeci F, Mariscalco G, Ranocchi F, et al. Transareolar robotic-assisted access to the mitral valve. Innovations 2015; 10 438-40.

13. Cheng DC, Martin J, Lal A, et al. Minimally invasive versus conventional open mitral valve surgery: a meta-analysis and systematic review. Innovations 2011; 6: 84-103.

14. De Bonis M, Al-Attar N, Antunes M, et al. Surgical and interventional management of mitral valve regurgitation: a position statement from the European Society of Cardiology working groups on cardiovascular surgery and valvular heart disease. Eur Heart J 2016; 37: 133-9.

15. von Oppell UO, Mohr FW. Chordal replacement for both minimally invasive and conventional mitral valve surgery using premeasured Gore-Tex loops. Ann Thorac Surg 2000; 70: 2166-8.

16. Goldstone AB, Atluri P, Szeto WY, et al. Minimally invasive approach provides at least equivalent results for surgical correction of mitral regurgitation: a propensity-matched comparison. J Thorac Cardiovasc Surg 2013; 145: 748-56.

17. Falk V, Cheng DC, Martin J, et al. Minimally invasive versus open mitral valve surgery: a consensus statement of the International Society of Minimally Invasive Coronary Surgery (ISMICS) 2010. Innovations 2011; 6: 66-76.

18. Tang P, Onaitis M, Desai B, et al. Minithoracotomy versus sternotomy for mitral surgery in patients with chronic renal impair- ment: a propensity-matched study. Innovations (Phila) 2013; 8 : 325-31.

19. Onnasch JF, Schneider F, Falk V, et al. Minimally invasive approach for redo mitral valve surgery: a true benefit for the patient. J Card Surg 2002; 17: 14-9.

20. Glauber M, Miceli A, Canarutto D, et al. Early and long-term outcomes of minimally invasive mitral valve surgery through right minithoracotomy: a 10-year experience in 1604 patients. J Cardiothorac Surg 2015; 10: 181.

21. Davierwala PM, Seeburger J, Pfannmueller B, et al. Minimally invasive mitral valve surgery: "The Leipzig experience". Ann Cardiothorac Surg 2013; 2: 744-50.

22. Casselman FP, Van Slycke S, Wellens F, et al. Mitral valve surgery can now routinely be performed endoscopically. Circulation 2003; 108 Suppl 1: ||48-54.

23. Modi P, Chitwood WR Jr. Retrograde femoral arterial perfusion and stroke risk during minimally invasive mitral valve surgery: is there cause for concern? Ann Cardiothorac Surg 2013; 2: E1.

24. Kowalewski M, Malvindi PG, Suwalski P, et al. Clinical safety and effectiveness of endoaortic as compared to transthoracic clamp for small thoracotomy mitral valve surgery: meta-analysis of observational studies. Ann Thorac Surg 2017; 103: 676-86.

25. Colak Z, Borojevic M, Bogovic A, et al. Influence of intraoperative cerebral oximetry monitoring on neurocognitive function after coronary artery bypass surgery: a randomized, prospective study. Eur J Cardiothorac Surg 2015; 47: 447-54.

26. Garbade J, Davierwala P, Seeburger J, et al. Myocardial protection during minimally invasive mitral valve surgery: strategies and cardioplegic solutions. Ann Cardiothorac Surg 2013; 2: 803-8.

27. Stammers AH, Miller R, Francis SG, et al. Goal-directed perfusion methodology for determining oxygenator performance during clinical cardiopulmonary bypass. J Extra Corpor Technol 2017; 49: 81-92.

28. Lenkin Al, Zaharov VI, Lenkin PI, et al. Normothermic cardiopulmonary bypass increases cerebral tissue oxygenation during combined valve surgery: a single-centre, randomized trial. Interact Cardiovasc Thorac Surg 2013; 16: 595-601.

29. Dass C, Simpson SA, Steiner RM, Guy TS. Preprocedural computed tomography evaluation for minimally invasive mitral valve surgery: what the surgeon needs to know. J Thorac Imaging 2015; 30: 386-96.

30. Cao C, Wolfenden H, Liou K, et al. A meta-analysis of robotic vs. conventional mitral valve surgery. Ann Cardiothorac Surg 2015; 4: 305-14.

31. Gammie JS, Zhao Y, Peterson ED, et al. Maxwell chamberlain memorial paper for adult cardiac surgery. Less-invasive mitral valve operations: trends and outcomes from the society of thoracic surgeons adult cardiac surgery database. Ann Thorac Surg 2010; 90: 1401-8.

Received: 13.09.2018, accepted: 24.10.2018 